\title{
Generación de doble banda en antenas de Microcinta rectangulares utilizando ranuras separadas en secuencia de cantor
}

\author{
Dual-Band generation in rectangular patch antennas using \\ separated slots based on cantor sequence
}

\author{
Andrés Hernández ${ }^{1 *} \quad$ Francisco López ${ }^{1} \quad$ Oscar Ossa $^{1}$ \\ Recibido 10 de octubre de 2017, aceptado 25 de marzo de 2019 \\ Received: October 10, 2017 Accepted: March 25, 2019
}

\begin{abstract}
RESUMEN
Un nuevo sistema de antenas de microcinta es presentado en este trabajo, en el cual se insertan ranuras rectangulares cuyas dimensiones y separación están determinadas por diferentes órdenes de la secuencia de Cantor. Las pérdidas por retorno fueron analizadas para determinar el efecto de este tipo de secuencia de ranuras en el parche, mostrando la generación de dos bandas en el rango de 1 a $6 \mathrm{GHz}$. La antena de microcinta fue diseñada aplicando un número de ranuras en el parche cuyos tamaños se basan en la secuencia Cantor hasta la cuarta iteración. El arreglo presentado permite tener dos frecuencias de resonancia en el rango de 1 a $6 \mathrm{GHz}$, los resultados computacionales y experimentales presentan una gran concordancia. La simulación de los modelos se realizó utilizando el software HFSS, utilizando un sustrato FR 4 con $\varepsilon_{r}=4,4$ y un espesor de $1,58 \mathrm{~mm}$. Las antenas presentadas en este artículo a diferencia de una antena de microcinta convencional con las mismas dimensiones, presenta dos bandas de frecuencia las cuales poseen ligeras diferencias entre cada iteración. Los nuevos sistemas de antenas tipo parche presentados en este trabajo permitieron obtener la generación de doble banda después de añadir ranuras con base en la secuencia de Cantor.
\end{abstract}

Palabras clave: Secuencia de Cantor, antena, microcinta, doble banda.

\begin{abstract}
A new microstrip antenna system is presented in this work, in which are inserted rectangular slots and different orders in the cantor sequence determine their dimension and separation. The return losses are analyzed to determine the effect of this type of slot sequence on the patch, showing the generation of dual-band behavior in the range of 1 to $6 \mathrm{GHz}$. The microstrip antenna is designed by several slots on the patch whose sizes are based on the Cantor sequence up to the fourth iteration. The presented arrangement allows having two resonance frequencies in the range of 1 to $6 \mathrm{GHz}$, the experimental results and the computer simulations show a high concordance. The simulation was performed using the HFSS software, using an FR4 substrate with $\varepsilon_{r}=4.4$ and a thickness of $1.58 \mathrm{~mm}$. The antennas presented in this article, unlike a conventional microstrip antenna with the same dimensions, present two bands of frequency, which have slight differences between each iteration. The new microstrip antenna systems presented in this work allowed to obtain a dual-band generation after adding slots based on the Cantor sequence.
\end{abstract}

Keywords: Cantor sequence, antenna, microstrip, dual band.

\footnotetext{
1 Facultad de Ingenierías / Instituto Tecnológico Metropolitano. Calle 54A No 30-01, Barrio Boston. Medellín, Colombia. E-mail: andreshernandez118627@correo.itm.edu.co; franciscolopez@itm.edu.co; okr.ossa@gmail.com

* Autor de correspondencia: andreshernandez118627@correo.itm.edu.co
} 


\section{INTRODUCCIÓN}

En la actualidad se han desarrollado una gran cantidad de trabajos que utilizan diferentes formas de ranuras sobre muy variadas geometrías de antenas de microcinta [1-4]; con el objetivo de modificar los parámetros característicos de dichas antenas. Algunas de estas ranuras se basan en geometrías en forma de $\mathrm{U}$ o en forma de $\mathrm{V}$ [5-7], ya que estas permiten altas ganancias y aumentar el ancho de banda, con la ventaja de ser diseños de estructuras simples. Particularmente, en [8] se explora de manera analítica los cambios generados en una antena de microcinta rectangular mediante la adición de una ranura en forma de $\mathrm{U}$ (frecuencias de resonancia y propiedades de radiación) sobre el parche y se indaga sobre la relación que puede tener la geometría del sistema y su efecto sobre los parámetros característicos de la antena. En [9-10] se plantean estudios muy interesantes sobre la adición de ranuras sobre una antena de microcinta rectangular (RMA, por las siglas en inglés de Rectangular Microstrip Antenna), con alimentación coaxial. Donde los autores analizan el cambio en las frecuencias de resonancia con la adición de una sola ranura; además, variando el largo, ancho y posición de esta se estudia el efecto que tiene la geometría sobre las perdidas por retorno o parámetro (S11). Los diseños de antenas de microcinta con base en ranuras (slots) permiten tener varias bandas de operación, y cubren múltiples aplicaciones de comunicación inalámbrica como WiFi, GPS, radar, WiMax, WLAN, comunicación punto a punto, entre otras. En los últimos años este tipo de estructuras ha recibido especial atención por su aplicación en tecnologías de la última década como el WiGig y 5G [11-13]. La demanda actual referente a sistemas de comunicación inalámbrica es alta, y es necesario o se hace más cómodo que los sistemas tiendan a disminuir en tamaño y aumentar en eficiencia y rendimiento, varios modelos han sido propuestos con el objetivo de lograr esta reducción de tamaño [14-16]. Por otra parte, con el objetivo de aumentar la eficiencia del sistema, se han propuesto técnicas basadas en la inclusión de metamateriales [17-18], resonadores en forma de anillo [19-20], geometrías fractales [21] o el uso de distintos sustratos dieléctricos ya sea variando $\varepsilon_{r}, \mu_{r}$, o el espesor del sustrato.

El estudio de antenas con geometrías fractales ha arrojado importantes resultados y aplicaciones en el diseño de antenas, los trabajos preliminares [22-25] han sido ampliamente referenciados y se ha evidenciado el impacto que tiene en la generación de múltiple banda la utilización de estructuras fractales en antenas. La secuencia de Cantor es uno los fractales geométricos clásicos, inicialmente fue propuesto por Georg Cantor como una solución a los problemas del continuo, que hoy en día es estudiada para el diseño de sistemas de baja dimensión como las antenas de microcinta [26], sistemas de banda ancha, antenas de múltiple banda, y su posible aplicación en sistemas de comunicación inalámbrica. La secuencia de Cantor posee una definición geométrica que elimina en cada iteración el segmento abierto correspondiente al tercio central de cada intervalo, es decir se trata de un segmento de longitud fija al que se divide en tres partes, en el cual se suprime el tercio de segmento central. Este procedimiento se repite en los segmentos que resultan de cada división. Como se ve, es un procedimiento recursivo que es siempre el mismo independientemente del nivel de construcción en él que se encuentre; por tanto se trata de lo que se ha definido como fractal. Hoy en día se pueden encontrar variedad de diseños de antenas de microcinta con modificaciones en su geometría obedeciendo a geometrías fractales como la curva de Koch [27], la secuencia de Cantor, la alfombra de Sierspinky, combinaciones de distintas geometrías fractales, entre otras estructuras similares [28-30] cuyo factor común es la repetición de un objeto o estructura básica en diferentes escalas, cada una generando diferentes cambios en el sistema de modo que permitan optimizar y, en ciertas ocasiones, reducir el tamaño del sistema de comunicación. Las geometrías fractales aplicadas sobre los diseños de las antenas tienen en común que aportan características de múltiple banda, ofreciendo buenas ganancias o menores perdidas en dos o más frecuencias de operación; motivo que las hace de especial utilidad para aplicaciones en redes inalámbricas.

Existen varias formas de obtener dos bandas en una antena tipo parche, en general se puede clasificar en tres formas [31]: utilizando la primera resonancia asociada a cada una de las dos dimensiones del parche rectangular, mediante la utilización de múltiples parches sobre el mismo plano o en diferentes planos y mediante la utilización de cargas reactivas como puntas, muescas, cortocircuitos, perforaciones, capacitores y ranuras. 
En este artículo presentamos un grupo de antenas de doble banda, diseñadas con base a un conjunto de ranuras cuyos tamaños se rigen por la secuencia de Cantor. Dado que la adición de ranuras a una antena tipo parche es una forma de generar doble banda, se parte de este resultado para proponer una secuencia de ranuras sobre el parche. La secuencia seleccionada es de tipo Cantor, la cual podría arrojar resultados interesantes en la generación de nuevas bandas de operación como se ha observado en otras formas fractales utilizadas en antenas de microcinta [22-25]. Primero se realizó la simulación del sistema sin ningún tipo de ranura, para identificar los parámetros característicos de la antena original; en busca de modificar los parámetros característicos de la antena adicionando ranuras, se realizaron diseños con base a 4 iteraciones de la secuencia de Cantor, notando cambios relevantes en el parámetro S11. Las antenas propuestas alcanzan fácilmente la operación de doble banda cuando se aplican sobre ellas un conjunto de ranuras cuyo tamaño se rige por la secuencia de Cantor. El diseño se realizó mediante un sustrato FR4 con $\varepsilon_{r}=4,4$ y una línea de transmisión acoplada a $50 \Omega$, todas las simulaciones se realizaron mediante el software comercial HFSS.

\section{DISEÑO}

Para el diseño general del sistema de antena de microcinta sobre el que se trabajaron los diferentes modelos con base en la secuencia de Cantor, se tomó un área de $17,84 \mathrm{~mm}$ por $17,37 \mathrm{~mm}$. Las cuatro iteraciones diferentes de la secuencia se realizaron teniendo en cuenta que el tamaño de las ranuras en la cuarta iteración (las de menor tamaño) no fueran menores que $120 \mu \mathrm{m}$ (pensando en la fabricación de la misma). También, se optó por obtener frecuencias resultantes en los diferentes parámetros $\mathrm{S} 11$ dentro del rango de $1 \mathrm{GHz}$ a $6 \mathrm{GHz}$, esto con el fin de poder realizar la componente experimental. En cuanto al diseño de la antena original, la relación que describe la frecuencia de resonancia para el modo fundamental, está dada por la ecuación (1).

$$
f r_{010}=\frac{c}{2\left(L_{\text {eff }}\right) \sqrt{\varepsilon_{\text {reff }}}}
$$

Donde $L_{\text {eff }}$ y $\varepsilon_{\text {reff }}$ son la longitud y constante dieléctrica efectiva de la antena, respectivamente [32]. El diseño esquemático de la antena de microcinta original es el mostrado en la Figura 1. El diseño consta de una placa de sustrato dieléctrico cuyo material es FR4, el cual es comúnmente utilizado como base de muchos dispositivos electrónicos y circuitos integrados. En la parte superior del sustrato, se tiene la antena de microcinta y la línea de transmisión con impedancia de $50 \Omega$, y en la parte posterior, el plano a tierra de la antena.

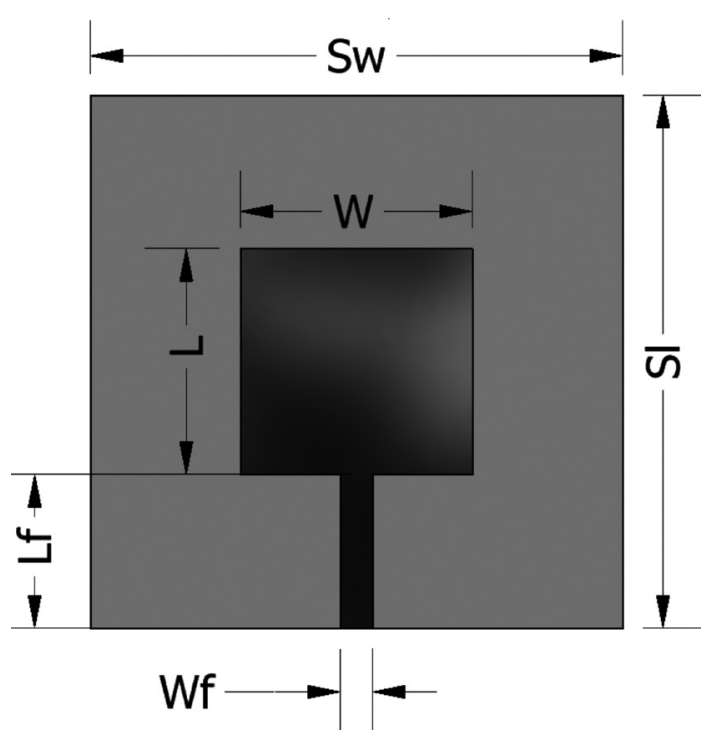

Figura 1. Antena Original con parámetros de diseño: $L=17.37, W=17.84, S l=41$, $S w=41, L f=11.82$ y $W f=2.5$; todas las medidas en mm. La lámina de FR4 usada para el diseño de la antena tiene un espesor de $1.58 \mathrm{~mm}$.

\section{CONFIGURACION DE LAS ANTENAS}

Para los modelos de antenas con base en la secuencia de Cantor y el diseño planteado en la Figura 1, se definió un espaciamiento de $0.36 \mathrm{~mm}$ entre los bordes de las ranuras y los bordes del parche (ambos paralelos a $L$ ), además, del total de la longitud del parche se escogió un tramo igual a $12,96 \mathrm{~mm}$, para trabajar en las iteraciones de la secuencia de Cantor. Con base en la anterior delimitación, se itero la secuencia de Cantor y se dispuso las ranuras sobre el parche de la antena, tal como lo muestra la Figura 2; donde, a medida que aumenta el orden de Cantor, los tercios centrales de cada división se rellenan con cobre. Como se puede apreciar en la Figura 2 el número de ranuras aumenta a medida que aumenta el orden de la secuencia, la cantidad 

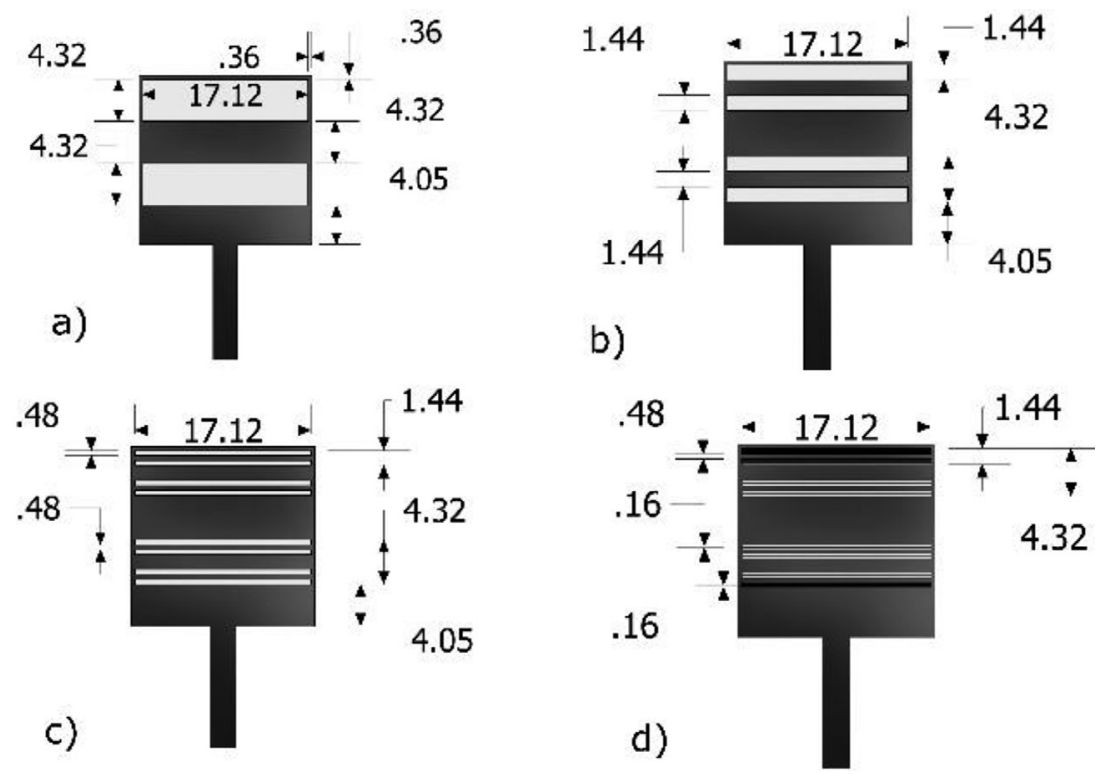

Figura 2. Diseño Esquemático de las antenas en sus cuatro iteraciones. a) Cantor Orden 1, b) Cantor Orden 2, c) Cantor Orden 3 y d) Cantor Orden 4.

de ranuras por secuencia aumenta en un orden de base dos, de manera que se tienen $2^{1}$ ranuras para la primer iteración, y $2^{4}$ ranuras para la última iteración analizada, correspondiente al orden 4 ; respecto al tamaño de las ranuras estos disminuyen por un factor de $1 / 3$ a medida que aumenta cada iteración, en la Tabla 1 se pueden apreciar la cantidad de ranuras por iteración, así como su tamaño.

\section{SIMULACIÓN}

La simulación del sistema se realizó usando Ansoft High Frequency Structure Simulator (HFSS). Se obtuvieron los resultados del parámetro S11 de cada modelo con base en la secuencia de Cantor, así como para la antena original (sin ninguna ranura). El objetivo de la simulación apunta al análisis de las frecuencias de resonancia de las antenas cuando se aplica sobre ellas un conjunto de ranuras según esta secuencia. Se evidencia que el coeficiente de reflexión se ve desplazado en frecuencia y aumenta su magnitud con tan solo agregar ranuras de Cantor en orden 1 . Teniendo en cuenta que cuando se aplica este método en el diseño, se generan dos frecuencias de resonancia alrededor de los 3,7 GHz y 4,5 GHz.

\section{RESULTADOS}

Varios modelos de antenas de microcinta con base a diferentes combinaciones de la secuencia de Cantor fueron diseñados en este artículo. Posterior a la simulación de los sistemas, se construyeron los prototipos y realizaron las medidas del parámetro S11 usando un VNA (Vector Network Analyzer - R\&S FSH8). Los resultados computacionales y experimentales son mostrados en la Figura 4, donde se puede observar gran similitud en las frecuencias de resonancia de cada antena las cuales están alrededor de 3,7 y 4,5 GHz, exceptuando el modelo de orden 1 cuya única frecuencia se encuentra alrededor de 3,7 GHz. En la Tabla 2 se puede apreciar las frecuencias de resonancia para cada modelo y el ancho de banda para la segunda frecuencia; los resultados experimentales muestran una buena

Tabla 1. Dimensiones de la antena de microcinta.

\begin{tabular}{|l|c|c|c|c|}
\hline \multicolumn{1}{|c|}{ Iteración } & Cantor orden 1 & Cantor orden 2 & Cantor orden 3 & Cantor orden 4 \\
\hline Cantidad de ranuras & $2^{1}$ & $2^{2}$ & $2^{3}$ & $2^{4}$ \\
\hline Tamaño de las ranuras & $4.32 \mathrm{~mm}$ & $1.44 \mathrm{~mm}$ & $0.48 \mathrm{~mm}$ & $0.16 \mathrm{~mm}$ \\
\hline
\end{tabular}




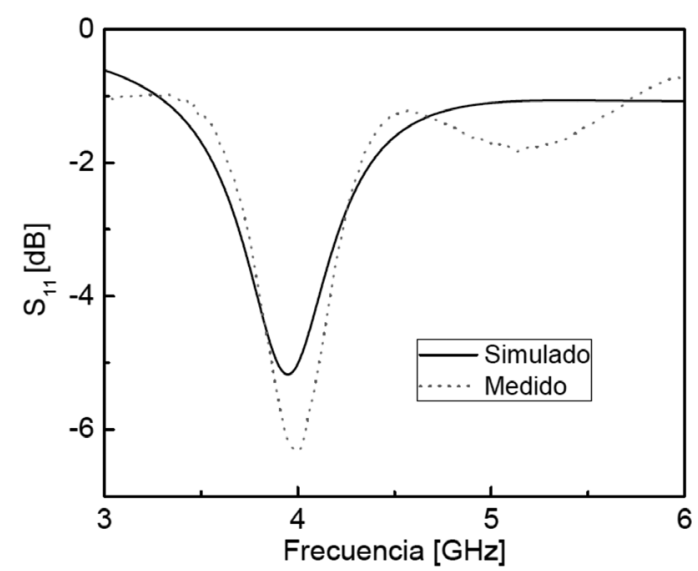

Figura 3. Parámetro S11 simulado y medido para antena sin ranuras (véase Figura 1).

Tabla 2. Frecuencias de resonancia y anchos de banda.

\begin{tabular}{|l|c|c|c|c|}
\hline \multicolumn{1}{|c|}{ Iteración } & $\mathbf{C 1}$ & $\mathbf{C 2}$ & $\mathbf{C 3}$ & $\mathbf{C 4}$ \\
\hline $\begin{array}{l}\text { Frecuencia de } \\
\text { resonancia 1 }\end{array}$ & $3,7 \mathrm{GHz}$ & $3,7 \mathrm{GHz}$ & $3,7 \mathrm{GHz}$ & $3,7 \mathrm{GHz}$ \\
\hline $\begin{array}{l}\text { Frecuencia de } \\
\text { resonancia 2 }\end{array}$ & $\mathrm{NA}$ & $4,5 \mathrm{GHz}$ & $4,5 \mathrm{GHz}$ & $4,5 \mathrm{GHz}$ \\
\hline $\begin{array}{l}\text { Ancho de } \\
\text { banda }\end{array}$ & $50 \mathrm{MHz}$ & $50 \mathrm{MHz}$ & $20 \mathrm{MHz}$ & $50 \mathrm{MHz}$ \\
\hline
\end{tabular}

aproximación a lo esperado con los resultados computacionales; en la Figura 3 se observa que el mínimo coeficiente de reflexión para la antena sin ranuras se da en $3,9 \mathrm{GHz}$ pero este pico no alcanza a cumplir el criterio $\leq-10 \mathrm{db}$, por lo tanto no presenta una condición adecuada para su uso en la transmisión de algún tipo de señal. Por el contrario el coeficiente de reflexión de cada modelo en base a la secuencia de Cantor (Figura 4) es mucho mejor que -10db, lo que indica que al realizar la modificación en la geometría del parche por medio de ranuras cuyos tamaño se rigen por esta secuencia, permiten mejorar el sistema, presentando dos frecuencias de resonancia en cualquiera de las combinaciones, produciendo mejores condiciones para la operación de las antenas. Los resultados obtenidos a partir del modelo de Cantor orden 1 para ambas frecuencias de resonancia en 3,7 y $4,5 \mathrm{GHz}$, permiten notar con claridad que esta frecuencias también aparecen en el resto de modelos, presentando leves diferencias respecto a las perdidas, indicando un mejor acople debido a la geometría del modelo de orden 4.
La discrepancia entre los resultados obtenidos mediantes simulaciones y medidas experimentales, se deben en gran medida al proceso de fabricación, soldado y acople.

Las antenas diseñadas en este trabajo alcanzan su primer modo de resonancia alrededor de los $3,7 \mathrm{GHz}$, teniendo en cuenta que la frecuencia de resonancia fundamental para una antena con las mismas dimensiones sin adición de ranuras en secuencia de Cantor se encuentra alrededor de los 3,9 GHz, lo que quiere decir que el área ocupada por las nuevas antenas es un $89 \%$ de la original, indicando que el área total de las antenas propuestas pueden ser reducidas en un $11 \%$, algo que sustancialmente podría aportar a la reducción total de un sistema de comunicaciones con base a la secuencia de Cantor.

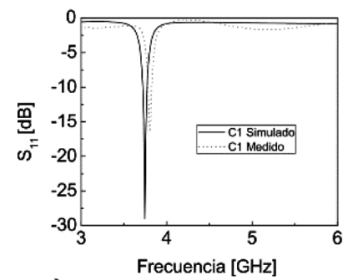

a)

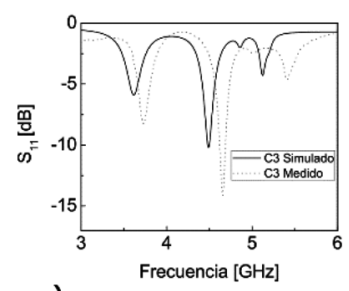

c)

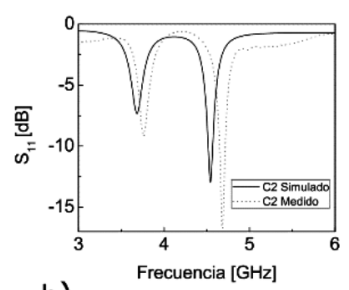

b)

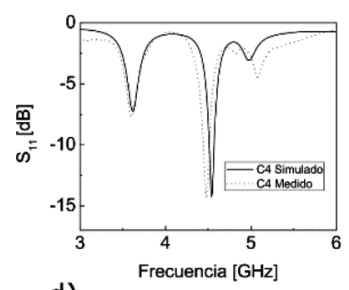

d)
Figura 4. Parámetro S11 simulado y medido para las antenas en cuatro órdenes de la secuencia de Cantor. a) Cantor Orden 1, b) Cantor Orden 2, c) Cantor Orden 3 y d) Cantor Orden 4.

Como se ha podido evidenciar la inclusión de la secuencia de Cantor sobre el sistema de antena de microcinta planteado inicialmente en este trabajo, permite que se generen dos bandas de frecuencia (Dual-Band), las cuales pertenecen a la banda SHF del espectro radioeléctrico; podemos decir a partir de estos resultados que el sistema mejora respecto a sus condiciones iniciales debido a la creación de nuevas frecuencias de resonancia las cuales 
juegan un papel muy importante ya que partiendo de un modelo que no posee condiciones óptimas, se llega a varios que con las mismas dimensiones aumentan la eficiencia, desempeño y el número de aplicaciones donde podría funcionar este tipo de sistemas. Estos sistemas con base a la secuencia de Cantor pueden ser adecuados para aplicaciones de radiocomunicación por trayectoria directa o línea de vista, sistemas satélites, sistemas de microondas, WiMAX, redes de área personal, y redes WLAN.

Es importante mencionar que al practicar diferentes tipos de ranuras sobre la capa radiante se han observado la aparición de nuevos lóbulos en el patrón de radiación [33], modificación en algunas propiedades de la polarización, tales como polarización dual [34] y polarización circular [35], por mencionar algunas.

\section{DISCUSIÓN}

Al estudiar el coeficiente de reflexión de la antena en la ventana de frecuencia analizada, antes de realizar modificaciones en la capa radiante, la frecuencia de resonancia corresponde al modo dominante $\mathrm{TM}_{010}$ con un valor aproximado de $4 \mathrm{GHz}$ y, el cual es coherente comparado con el resultado del modelo simulado. La condición de resonancia para el modo fundamental está gobernada por el parámetro de longitud L del parche radiante de la antena y corresponde a la frecuencia de resonancia de menor valor. En la frecuencia fundamental, la distribución de densidad de corriente tiene una dirección paralela a la longitud del parche radiante de la antena y, debido a que esta es continua en toda la superficie del parche (además de que se cumple $h \ll \lambda$ ), el campo eléctrico es paralelo a las capas conductoras y se distribuye en dos semiciclos de onda a lo largo de la longitud del parche. En el momento de realizar modificaciones en el diseño de la antena con el arreglo de ranura de primer orden (C1) de la serie de Cantor, es evidente que la frecuencia de resonancia fundamental toma un valor inferior; esto se atribuye a un aumento en la longitud eléctrica del parche de la antena debido al cambio en la distribución de la corriente causado por la discontinuidad que representan las ranuras. En consecuencia, el parche de la antena se

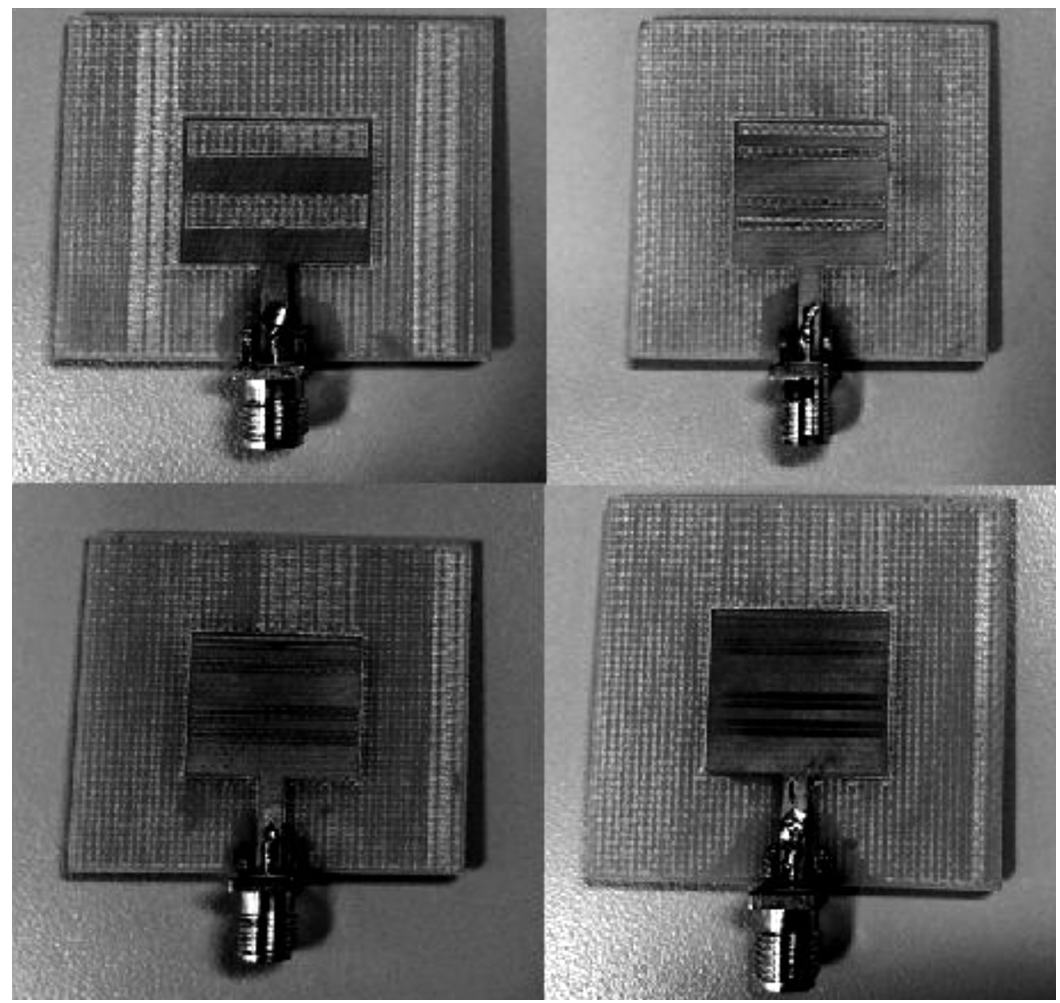

Figura 5. Antenas construidas (C1, C2, C3 y C4). 
percibe eléctricamente más largo y la frecuencia de resonancia tiende a un valor inferior, lo que es acorde con el modelo de análisis de líneas de transmisión. Teniendo en cuenta la relación entre la longitud y la frecuencia de resonancia, es válido hacer la observación de que esta última se ve saturada por el infinitésimo espesor de las ranuras, la velocidad de aumento de la longitud del fractal es mayor que la de la disminución de la frecuencia de resonancia, tal como se confirma en [36]. En otras palabras, el espesor de las ranuras y la separación entre ellas tienden a tomar valores muy pequeños a medida que aumenta el orden del fractal, y la primera frecuencia de resonancia no percibe estas variaciones, lo que ocasiona la saturación de esta frecuencia y forma una asíntota o tendencia a un valor constante a medida que aumenta el orden del fractal. Por otro lado, las modificaciones en el diseño de la antena realizadas en base a la serie de Cantor ocasionan un cambio en el patrón del campo dentro de la antena (cavidad), donde, el campo en el modo fundamental que se configuraba en dos semiciclos a lo largo de $L$ es perturbado, dando lugar a más oscilaciones a lo largo del parche, tal como se muestra en Figura 6 para el campo en la componente $z$. Además, debido a las ranuras (discontinuidades) el campo eléctrico presenta componentes en diferentes dirección a $z$, en contraste a su comportamiento para la antena sin ninguna modificación. En la Figura 6, se muestra las oscilaciones del campo en la componente $z$ a medida que aumenta el orden de la secuencia de Cantor, la figura pretende describir como se modifican las oscilaciones del campo en dicha componente a medida que se adicionan ranuras a la antena y hacer un contraste con el modo fundamental donde solo aparecen dos semiciclos de onda. Finalmente, dos características eléctricas que se modifican debido al fractal son el acople de la impedancia de la antena y el ancho de banda de impedancia. Para la primera, se aprecia que el diseño de la iteración C1 tiene el mejor acople, con pérdidas de potencia en reflexión menores a los $-15 d B$, lo cual es un valor aceptable para muchas aplicaciones de comunicaciones inalámbricas. En cuanto al ancho de banda de impedancia, se observa una disminución debido a la modificación de la antena con la adición de las ranuras, lo cual es un resultado esperado de la aplicación de fractales en antenas.

Es claro que bajo estos modelos las antenas conservan dos propiedades fundamentales de una estructura fractal, geometría y frecuencias de resonancia. La primera, parte del concepto visual de que una estructura fractal es auto similar, es decir, las antenas están constituidas de copias de diferente tamaño de una misma figura (ranura), por más que estas se repitan en el espacio, están destinadas a conformar la unidad original de la que provienen. Por otra parte las frecuencias de resonancia de las antenas aumentan respecto al modelo inicial, y se mantienen a medida que se aumentan las iteraciones, lo cual es una de las propiedades más importantes de las geometrías fractales aplicadas a antenas, el generar dos o más frecuencias de resonancia (multibanda).

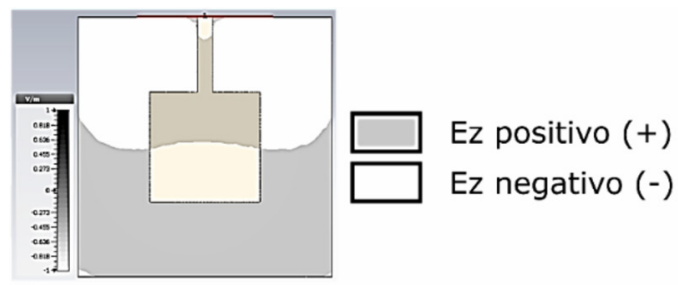

a)

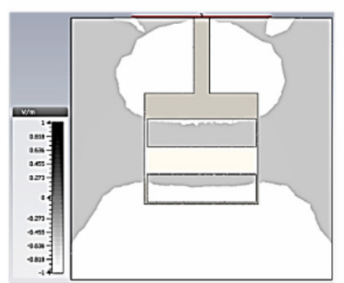

b)

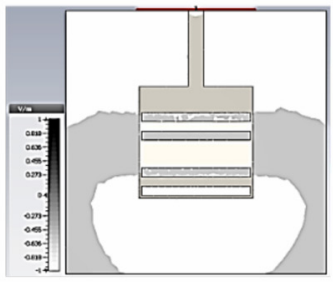

c)

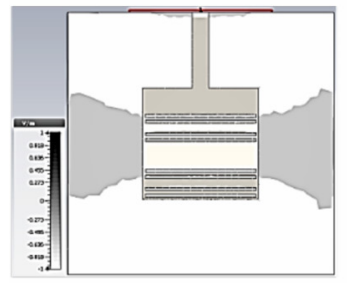

d)

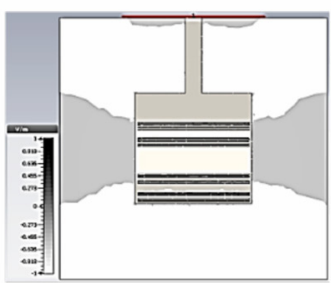

e)

Figura 6. Configuración del campo eléctrico en el eje $z$ (plano $x-y$ ) para los diferentes diseños de antenas: a) parche sin modificaciones $\mathrm{y}$ frecuencia de resonancia fundamental $\left(T M_{010}\right)$. Escala normalizada con $6.66 e^{4} v / \mathrm{m}$. b) secuencia de Cantor orden $\mathrm{C} 1$ y escala normalizada con $2.5 e^{5} \mathrm{v} / \mathrm{m}$ c) secuencia de Cantor orden $\mathrm{C} 2$ y escala normalizada con $1.1 e^{5} \mathrm{v} / \mathrm{m}$, d) secuencia de Cantor orden $\mathrm{C} 3$ y escala normalizada con $1.05 e^{5} \mathrm{v} / \mathrm{m}$ y e) secuencia de Cantor orden C4 y escala normalizada con $4.72 e^{5} \mathrm{v} / \mathrm{m}$. 


\section{CONCLUSIONES}

Nuevos modelos de antenas de microcinta diseñadas con una geometría de múltiples ranuras en el parche según la secuencia de Cantor fueron propuestos en este artículo. Un sustrato FR4 con $\varepsilon_{r}=4.4$ es usado para construir las antenas, se logra una buena concordancia entre los resultados experimentales y los computacionales. Es importante mencionar que para precisar muy bien las frecuencias centrales de operación, se requiere una alta precisión en la fabricación de las antenas, pues las mismas presentan anchos de banda reducidos. La inclusión de la secuencia de Cantor provee dos frecuencias de resonancia $(3,7 \mathrm{GHz}$ y $4,5 \mathrm{GHz})$ en cualquiera de las iteraciones analizadas en este trabajo, haciendo el sistema apto para sistemas y tecnologías de comunicación inalámbrica. Es importante resaltar que gracias a la adición de ranuras en secuencia de Cantor se puede mejorar el desempeño de una antena simple, que en su estado inicial no presenta las condiciones adecuadas para su uso en algún tipo de sistema de comunicación inalámbrica, e inmediatamente se adicionan ranuras con base en la secuencia, el sistema incrementa el número de frecuencias de resonancia y disminuye las perdidas en estas, por lo que se plantea una relación directa entre el diseño con base a ranuras que se rigen por la secuencia de Cantor y el impacto que este genera en el coeficiente de reflexión.

\section{TRABAJOS FUTUROS}

Se dará continuidad al estudio de ranuras en secuencia de Cantor teniendo en cuenta la variación de otros parámetros, como pueden ser la longitud y dirección de las ranuras, lo cual podría generar desplazamientos en las bandas, siendo útil para antenas que requieran funcionar en dos bandas prefijadas. Es de observar que si bien el nivel de adaptación no alcanza el valor estándar de $10 \mathrm{~dB}$ para algunas bandas, si se logra identificar el valor de la resonancia. En trabajos futuros se explorarán algunos métodos propuestos en la literatura para mejorar la adaptación en las antenas tipo parche conectadas de forma directa a la línea de alimentación.

\section{AGRADECIMIENTOS}

Este trabajo fue apoyado gracias al Instituto Tecnológico metropolitano y la beca pasantía Jóvenes
Investigadores e Innovadores Colciencias en alianza ITM. Se agradece a todos los implicados en el trabajo así como las contribuciones y revisiones realizadas.

\section{REFERENCIAS}

[1] J. Chen, K.F. Tong, A. Al-Armaghany and J. Wang. "A dual-band dual- polarization slot patch antenna for gps and wi-fi applications". IEEE Antennas and Wireless Propagation Letters. Vol. 15, pp. 406-409. 2016.

[2] M.C. Bhad, V.G. Kasabegoudar and M. P. Rodge. "Electrically small rectangular patch antenna with slot for mimo applications". Wireless and Mobile Technologies. Vol. 1 $\mathrm{N}^{\circ}$ 1, pp. 25-28. 2013.

[3] H.A. Majid, M.K.A. Rahim, M.R. Hamid, N.A. Murad and M.F. Ismail. "Frequencyreconfigurable microstrip patch-slot antenna". IEEE Antennas and Wireless Propagation Letters. Vol. 12, pp. 218-220. 2013.

[4] H. Elsadek and D.M. Nashaat, "Multiband and uwb v-shaped antenna configuration for wireless communications applications". IEEE Antennas and Wireless Propagation Letters. Vol. 7, pp. 89-91. 2008.

[5] S. Liu, W. Wu and D.G. Fang. "Single-feed dual-layer dual-band e- shaped and u-slot patch antenna for wireless communication application". IEEE Antennas and Wireless Propagation Letters. Vol. 15, pp. 468-471. 2016.

[6] S.L.S. Yang, A.A. Kishk and K.F. Lee. "Frequency reconfigurable u-slot microstrip patch antenna". IEEE Antennas and Wireless Propagation Letters. Vol. 7, pp. 127-129. 2008.

[7] M. Rostamzadeh, S. Mohamadi, J. Nourinia, C. Ghobadi and M. Ojaroudi. "Square monopole antenna for uwb applications with novel rod-shaped parasitic structures and novel v-shaped slots in the ground plane". IEEE Antennas and Wireless Propagation Letters. Vol. 11, pp. 446-449. 2012.

[8] S. Weigand, G.H. Huff, K.H. Pan and J.T. Bernhard. "Analysis and design of broad-band single-layer rectangular u-slot microstrip patch antennas". IEEE Transactions on Antennas and Propagation. Vol. $51 \mathrm{~N}^{\mathrm{o}} 3$, pp. 457-468. March 2003.

[9] M. Joler and J. Kucan. "Impact of slot parameters on the three resonant frequencies 
of a rectangular microstrip antenna: Study of the impact of the slot length, width, and position". IEEE Antennas and Propagation Magazine. Vol. $57 \mathrm{~N}^{\circ}$ 4, pp. 48-63, Aug, 2015.

[10] O.D.O. Molina, A.L.F. Camen, C.E. Ramirez, E.E.R. Vera and F.E.L. Giraldo. "Efectos en el coeficiente de reflexión de una antena de microcinta rectangular debidos a las modificaciones de posición de una ranura en la capa radiante". Rev. EIA. Vol. $14 \mathrm{~N}^{\circ} 28$, pp. 85-97. 2018.

[11] H. Mopidevi, H.V. Hunerli, E. Cagatay, N. Biyikli, M. Imbert, J. Romeu. L. Jofre and B.A. Cetiner. "Three-dimensional microfabricated broadband patch antenna for wigig applications". IEEE Antennas and Wireless Propagation Letters. Vol. 13, pp. 828-831. 2014.

[12] D. Wang and C.H. Chan. "Multiband antenna for wifi and wigig communications". IEEE Antennas and Wireless Propagation Letters. Vol. 15, pp. 309-312. 2016.

[13] J.S. Park, J.B. Ko, H.K. Kwon, B.S. Kang, B. Park and D. Kim. "A tilted combined beam antenna for $5 \mathrm{~g}$ communications using a 28-ghz band". IEEE Antennas and Wireless Propagation Letters. Vol. 15, pp. 1685-1688. 2016.

[14] S.I.H. Shah, S. Bashir and A. Altaf. "Miniaturization of microstrip patch antenna by using various shaped slots for wireless communication systems". In 2014 XIXth International Seminar/Workshop on Direct and Inverse Problems of Electromagnetic and Acoustic Wave Theory (DIPED), pp. 92-95. Sept 2014.

[15] A. Motevasselian and W.G. Whittow, "Patch size reduction of rectangular microstrip antennas by means of a cuboid ridge". IET Microwaves, Antennas Propagation. Vol. 9 $\mathrm{N}^{\mathrm{o}}$ 15, pp. 1727-1732. 2015.

[16] K.Z. Rajab, R. Mittra and M.T. Lanagan. "Size reduction of microstrip patch antennas with left-handed transmission line loading". IET Microwaves, Antennas Propagation. Vol. $1 \mathrm{~N}^{\circ}$ 1, pp. 39-44. February, 2007.

[17] H. Zhou, Z. Pei, S. Qu, S. Zhang, J. Wang, Z. Duan, H. Ma and Z. Xu. "A novel highdirectivity microstrip patch antenna based on zero-index metamaterial". IEEE Antennas and Wireless Propagation Letters. Vol. 8, pp. 538-541. 2009.

[18] M.M. Islam, M.T. Islam, M. Samsuzzaman and M.R.I. Faruque. "Compact metamaterial antenna for uwb applications". Electronics Letters. Vol. 51 N $^{\circ} 16$, pp. 1222-1224. 2015.

[19] Ó. Quevedo-Teruel, M.N.M. Kehn and E. Rajo-Iglesias. "Dual-band patch antennas based on short-circuited split ring resonators". IEEE Trans. Antennas Propag. Vol. $59 \mathrm{~N}^{\circ} 8$, pp. 2758-2765. 2011.

[20] Y.H. Ren, J. Ding, C.J. Guo, Y. Qu and Y.C. Song. "A wideband dual-polarized printed antenna based on complementary split-ring resonators". IEEE Antennas and Wireless Propagation Letters. Vol. 14, pp. 410-413. 2015.

[21] V.V. Reddy and N.V.S.N. Sarma. "Compact circularly polarized asymmetrical fractal boundary microstrip antenna for wireless applications". IEEE Antennas and Wireless Propagation Letters. Vol. 13, pp. 118-121. 2014.

[22] C. Puente, J. Romeu, R. Pous, X. Garcia and F. Benitez. "Fractal multiband antenna based on the Sierpinski gasket". Electronics Letters. Vol. $32 \mathrm{~N}^{\circ}$ 1, pp. 1-2. 1996.

[23] C. Puente-Baliarda and R. Pous. "Fractal design of multiband and low side-lobe arrays". IEEE Transactions on Antennas and Propagation. Vol. 44 N 5, p. 730. 1996.

[24] C. Puente, J. Romeu, R. Bartoleme and R. Pous. "Perturbation of the Sierpinski antenna to allocate operating bands". Electronics letters. Vol. $32 \mathrm{~N}^{\circ}$ 24, pp. 2186-2188. 1996.

[25] C. Puente-Baliarda, J. Romeu, R. Pous, A. Cardama. "On the behavior of the Sierpinski multiband fractal antenna". IEEE Transactions on Antennas and propagation. Vol. $46 \mathrm{~N}^{\circ} 4$, pp. 517-524. 1998.

[26] B. Manimegalai, S. Raju and V. Abhaikumar. "A multifractal Cantor an- tenna for multiband wireless applications". IEEE Antennas and Wireless Propagation Letters. Vol. 8, pp. 359362. 2009.

[27] J. Guterman, A.A. Moreira and C. Peixeiro. "Microstrip fractal antennas for multistandard terminals". IEEE Antennas and Wireless Propagation Letters. Vol. $3 \mathrm{~N}^{\circ} 1$, pp. 351354. Dec 2004.

[28] W.L. Chen, G.M. Wang and C.X. Zhang. "Small-size microstrip patch antennas 
combining koch and sierpinski fractal-shapes". IEEE Antennas and Wireless Propagation Letters. Vol. 7, pp. 738-741. 2008.

[29] H. Oraizi and S. Hedayati. "Miniaturized uwb monopole microstrip antenna design by the combination of giusepe peano and sierpinski carpet fractals". IEEE Antennas and Wireless Propagation Letters. Vol. 10, pp. 67-70. 2011.

[30] G. Liu, L. Xu and Z. Wu. "Dual-band microstrip rfid antenna with tree-like fractal structure". IEEE Antennas and Wireless Propagation Letters. Vol. 12, pp. 976-978. 2013.

[31] S. Maci and G. BifJi Gentili. "Dual-Frequency Patch Antennas". IEEE Antennas Propag. Mag. Vol. 39, Issue 6, pp. 13-20, Dec. 1997.

[32] C.A. Balanis. "Antenna theory: analysis and design”. MICROSTRIP ANTENNAS, third Ed. John Wiley Sons. 2005.
[33] S. Maci. "Dual-band slot-loaded patch antenna". IEEE Proceedings - Microwaves, Antennas and Propagation. Vol. 142, Issue 3, p. 225. 1995.

[34] K. Gosalia and G. Lazzi. "Reduced size, dual-polarized microstrip patch antenna for wireless communications". IEEE Transactions on Antennas and Propagation. Vol. $51 \mathrm{~N}^{\circ} 9$, pp. 2182-2186. 2003.

[35] H.L. Zhu, S.W. Cheung, K.L. Chung and T.I. Yuk. "Linear-to-Circular Polarization Conversion Using Metasurface". IEEE Transactions on Antennas and Propagation. Vol. $61 \mathrm{~N}^{\circ}$ 9, pp. 4615-4623. 2013.

[36] J.P. Gianvittorio and Y. Rahmat-Samii. "Fractal antennas: a novel antenna miniaturization technique, and applications". IEEE Antennas Propag. Mag. Vol. $44 \mathrm{~N}^{\mathrm{o}}$ 1, pp. 20-36, Feb. 2002. 